\title{
Dust coagulation in protoplanetary disks: A rapid depletion of small grains
}

\author{
C. P. Dullemond and C. Dominik \\ 1 Max Planck Institut für Astrophysik, PO Box 1317, 85741 Garching, Germany \\ e-mail: dullemon@mpia.de \\ 2 Sterrenkundig Instituut "Anton Pannekoek", Kruislaan 403, 1098 SJ Amsterdam, The Netherlands \\ e-mail: dominik@science.uva.nl
}

Received 28 September 2004 / Accepted 29 November 2004

\begin{abstract}
We model the process of dust coagulation in protoplanetary disks and calculate how it affects their observational appearance. Our model involves the detailed solution of the coagulation equation at every location in the disk. At regular time intervals we feed the resulting 3D dust distribution functions into a continuum radiative transfer code to obtain spectral energy distributions. We find that, even if only the very basic - and well understood - coagulation mechanisms are included, the process of grain growth is much too quick to be consistent with infrared observations of T Tauri disks. Small grains are removed so efficiently that, long before the disk reaches an age of $10^{6}$ years typical of T Tauri stars, the SED shows only very weak infrared excess. This is inconsistent with observed SEDs of most classical T Tauri stars. Small grains must be replenished, for instance by aggregate fragmentation through high-speed collisions. A very simplified calculation shows that when aggregate fragmentation is included, a quasi-stationary grain size distribution is obtained in which growth and fragmentation are in equilibrium. This quasi-stationary state may last $10^{6}$ years or even longer, depending on the circumstances in the disk, and may bring the time scales into the right regime. If this is indeed the case, or if other processes are responsible for the replenishment of small grains, then the typical grain sizes inferred from infrared spectral features of $T$ Tauri disks do not necessarily reflect the age of the system (small grains $\rightarrow$ young, larger grains $\rightarrow$ older), as is often proposed. Indeed, there is evidence reported in the literature that the typical inferred grain sizes do not correlate with the age of the star. Instead, it is more likely that the typical grain sizes found in T Tauri star (and Herbig Ae/Be star and Brown Dwarf) disks reflect the state of the disk in some more complicated way, e.g. the strength of the turbulence, the amount of dust mass transformed into planetesimals, the amount of gas lost via evaporation etc. A simple evolutionary scenario in which grains slowly grow from pristine $0.1 \mu \mathrm{m}$ grains to larger grains over a period of a few Myr is most likely incorrect.
\end{abstract}

Key words. accretion, accretion disks - stars: circumstellar matter - stars: formation - stars: pre-main sequence infrared: stars - ISM: dust, extinction

\section{Introduction}

The coagulation of dust grains in protoplanetary disks is believed to be the first stage of planet formation. The dust grains, inherited from the interstellar medium, are initially particles of submicron size. But as a result of the high densities in the disk, and due to processes such as Brownian motion, settling and turbulence, the dust grains quickly hit each other, and generally stick and form aggregates of ever increasing size. It is believed that the continuing growth of such grains/aggregates eventually leads to the formation of planetesimals, which, through gravitational interaction, coalesce to form the rocky cores of planets. The subsequent accretion of gas onto these cores, if they are massive enough, then leads to the formation of gaseous giant planets. This is known as the core accretion model for giant planet formation (Lissauer 1993).

A significant body of theoretical research has so far mostly focused on our own solar system, trying to explain the structure of the system as well as the relevant time scales known from geological and meteoritic research. Theoretical models are constrained for example by the fact that the core of Jupiter must have formed fast enough (within a few Myrs) to start accreting gas while this gas was still present in the disk, and to stop the Asteroid Belt from forming a planet. On the other hand, the spread in ages for some inclusions in meteorites (CAIs and Chondrules) shows that that the process of putting together planetesimals must take at least 4-6 Myrs (Brearley \& Jones 1998). The pioneering work of Weidenschilling (1977, 1980 , 1984) considered the main processes contributing to particle growth, i.e. the collisons between grains caused by Brownian motion, vertical settling, radial drift and turbulence. Many details in the process have since then been refined, such as the detailed physics of the dust sublayer forming at the midplane of the disk (Dubrulle et al. 1995), the trapping of particles in anti-cyclonic vortices (e.g. Klahr \& Henning 1997; Cuzzi et al. 1993), the influence of aggregate shape on the timescales (Weidenschilling 1997b), as well as theoretical (Dominik \& Tielens 1995, 1996, 1997) and experimental 
(e.g. Blum \& Wurm 2000) studies of the strength and shape of the aggregates formed.

One of the interesting results is a time-scale problem related to the radial drift of particles through the disk. Once a particle becomes $\mathrm{cm}$-sized or larger, it tends to decouple from the gas and move on Keplerian orbits around the star. But the gas in the disk rotates with a slightly sub-Keplerian speed around the star, due to the small radial pressure support within the disk. The velocity difference between the gas and the particle causes friction and removes angular momentum from the dust particle. This results in a systematic drift inward toward the star. The time scale for this radial drift can be relatively short, on the order of 100 years for $\mathrm{m}$-sized particles at $1 \mathrm{AU}$ (Weidenschilling 1977). Only once the particle has grown to kilometer size does this radial drift stop because the friction has decreased sufficiently. The question is therefore: how can we grow grains from $\mathrm{cm}$ to $\mathrm{km}$ size within only $10^{3}$ orbits? Normal grain coagulation processes may or may not be fast enough, but particles of such large size have poor sticking properties. So the basic issue of grain coagulation is therefore how we can speed up coagulation in the models, and how can we enhance the sticking efficiency for larger particles.

A completely different set of constraints on grain growth in protoplanetary disks can be derived from the observations of circumstellar disks around young stars which are probably in the process of forming planets right now. The developments in infrared astronomy in recent years have opened up a new window on planet formation: the direct study of protoplanetary disks around T Tauri stars, Herbig Ae/Be stars and even around Brown Dwarfs. The ISO satellite, for instance, has provided infrared spectra of Herbig Ae/Be stars with unprecedented accuracy (e.g. Malfait et al. 1999; van den Ancker et al. 2000), giving clues to the composition and size of dust particles in these disks (Bouwman et al. 2000) and to the geometry of these disks (Meeus et al. 2001). The new Spitzer Space Telescope will do the same for T Tauri stars and Brown Dwarfs. With infrared interferometry (IOTA, PTI, VLTI, Keck etc.) much has been, and will be learned about the dust mineralogy and structure of the disk at sub-AU scales (e.g., Eisner 2003) and in the habitable zones around these stars (van Boekel et al. 2004a). All of these observations have drastically increased our knowledge of the physics of the birthplaces of planets. One of the main discoveries from (sub-)mm observations (e.g., Testi et al. 2003) and from mid-infrared spectroscopy (e.g., van Boekel et al. 2003) is the ample evidence for grain growth in disks. However, the information about grain growth is encoded in a complicated way in the data we can obtain. The particles seen by different observations are different in size, and are located at different locations in the disk. Extracting the desired constraints on grain growth processes therefore requires self-consistent modeling of the growth processes with disk structure and radiative transfer. For the earliest phases (collapse and early disk formation), this problem has been addressed by Suttner et al. (1999); Suttner \& Yorke (2001). However, most observed star+disk systems have already long evolved beyond this very early phase. Also, the process of the formation of planets may span up to 10 Myrs or more, which is much longer than the duration of the early disk phases studied by Suttner et al.
It is the purpose of this paper to compare self-consistent models of grain coagulation and settling in protoplanetary disks with the infrared observations of these objects. Interestingly, this has never been done before. In this paper we solve the coagulation equation (often referred to as the Smoluchowski equation), coupled to the equation for grain settling and vertical turbulent mixing. The coagulation equation evolves the dust size distribution in time, while the settling/mixing equation solves the vertical motion of the dust particles. At given time intervals we compute the spectral energy distribution (SED) and images of the disk using a $2 \mathrm{D}$ axisymmetric continuum radiative transfer code.

We will show in this paper that we are confronted with a new time scale problem, quite contrary to the one discussed above: we find that the coagulation of small particles is too fast to be consistent with infrared observations. From infrared spectroscopy in the 10 micron regime it seems that grain growth from $0.1 \mu \mathrm{m}$ to $2 \mu \mathrm{m}$ happens over a time scale of a few $10^{6}$ years (van Boekel et al. 2004b). We will show with our models that it is very difficult to prevent the complete depletion of grains up to $100 \mu \mathrm{m}$ within only $10^{4}$ years. We will suggest that aggregate fragmentation may provide a piece of the puzzle. In doing so, we will necessarily repeat computations done before for the solar system, but we will do so with better resolution and considering the observational consequences.

The structure of this paper is as follows: In Sect. 2 we describe the equations used to solve the problem of coagulation in a protoplanetary disk. From there we build our model step by step, adding the different processes one at a time, in order to show the relative importance, and to demonstrate the solidness of the main conclusion. In Sect. 3, we briefly discuss the fate of a single particle as it settles to the disk midplane and grows by sweeping up other grains. In Sect. 4 we solve the coagulationsettling equations for the entire ensemble of grains in a vertical slice in a disk, producing time-dependent grain size distribution functions as a function of height above the midplane. In Sect. 5 we use these local models to build a full disk model and to compute SEDs, with the conclusion that full coagulation depletes small grains much too rapidly to be consistent with infrared observations of disks. In Sect. 6 we introduce a possible remedy for this puzzle by showing that aggregate fragmentation might continuously replenish the small grains. In Sect. 7 we discuss our results.

\section{Equations}

The distribution of grains of a certain mass $m$, at a certain distance $R$ from the star, a certain height $Z$ above the midplane and at a time $t$ is given by the distribution function $f(R, Z, m, t)$. It is defined such that $f(R, Z, m, t) m \mathrm{~d} m$ is a dust mass density in $\mathrm{g} / \mathrm{cm}^{3}$. The dust grains undergo several processes. First of all, they settle toward the midplane, and at the same time they are turbulently stirred back up again. In the absence of coagulation, an equilibrium solution is eventually reached in which the upper layers of the disk are devoid of dust of a particular size, while below a certain $Z$ the grains are fully mixed with the gas and hence have more or less constant abundance (e.g., Dubrulle et al. 1995; Takeuchi \& Lin 2002). This process of settling and 
stirring has profound effects on the appearance of protoplanetary disks (Dullemond \& Dominik 2004b).

As the settling and turbulent stirring process takes place, the grains also coagulate. The relative motions between the dust grains, necessary for them to meet each other and form aggregates, are produced by various processes. In the models presented in this paper we include Brownian motion, differential settling and relative motions caused by turbulence. These are the most important processes for coagulation up to $\mathrm{cm}$ size particles, and we will describe in detail below how they are implemented in our model.

The problem of dust settling and stirring is a problem in the coordinate $Z$ while coagulation is a problem in the coordinate $m$. In principle they have to be solved simultaneously. In practice, the settling and mixing are solved in the same subroutine, and the coagulation in another subroutine. By using the technique of operator splitting one can switch between the two subroutines at each time step, thereby solving the simultaneous set of problems.

\subsection{Cross sections of particles}

An important quantity that will enter the equations is the cross section of a particle, for collisions with gas particles and with other particles. If we assume solid spheres, the collisional cross section for grain-gas collisions is simply given by $\sigma_{\mathrm{g}}=\pi a^{2}$ where $a$ is the radius of the particle. For collisions between two grains, the cross section will be $\sigma_{\mathrm{c}}=\pi\left(a_{1}+a_{2}\right)^{2}$. However, in reality, grains formed by aggregation are never compact spheres. The will have internal structure which depends on the formation mechanism. One extreme of the possibilities are Particle-Cluster Aggregates (PCA) which are formed when an aggregate grows by addition of small grains only. Such particles will tend to be spherical and porous, with a limiting porosity for large particles of about $90 \%$. At the other extreme are Cluster-Cluster-Aggregates (CCA) in which growth of an aggregate is dominated by accreting aggregates of its own size. Obviously, many other possibility exist, including a simultaneous mixture of PCA and CCA (growing by both small and large aggregates at the same time) and hierarchical growth by switching from one growth process to another, possibly several times. The purpose of this paper is not an in-depth study of these processes, which will be subject of a future paper. For the current study, we only use the three classical cases: compact, PCA and CCA particles. For each of these growth classes, a unique relation between mass and average collision cross sections can be derived. We here use the analytical fits by Ossenkopf (1993). These relations directly provide $\sigma_{\mathrm{g}}(m)$ and $\sigma_{\mathrm{c}}\left(m_{1}, m_{2}\right)$.

\subsection{Settling and vertical mixing}

The settling and mixing of grains is a 1D time-dependent problem in $z$, which has to be solved for each $R$ and $m$. We describe the settling and vertical mixing equations following the discussion of Dullemond \& Dominik (2004b). We refer to that paper for details. The equilibrium settling velocity for particles smaller than about $1 \mathrm{~cm}$ (Epstein regime) is:

$v_{\text {sett }}=-\frac{3 \Omega_{\mathrm{K}}^{2} z}{4 \rho c_{\mathrm{s}}} \frac{m}{\sigma_{\mathrm{g}}}$

where $\Omega_{\mathrm{K}} \equiv \sqrt{G M_{*} / R^{3}}$ is the Kepler rotational frequency, $c_{\mathrm{S}}$ is the isothermal sound speed. $\sigma_{\mathrm{g}}$ is the collisional cross-section of the dust grain for collisions with gas or very small dust particles, i.e. the projected surface of the grain, averaged over all directions.

The diffusion constant for turbulent mixing $D$ is given by

$D=\frac{\alpha_{\mathrm{turb}} c_{\mathrm{s}}^{2}}{\Omega_{\mathrm{K}} \mathrm{Sc}}$

where $\alpha_{\text {turb }}$ is the turbulence parameter. The symbol Sc is the Schmidt number defined as Sc $\equiv 1+$ St with the Stokes number St given by

$\mathrm{St}=\frac{3}{4} \frac{m}{\sigma_{\mathrm{g}}} \frac{\Omega_{\mathrm{K}}}{\rho c_{\mathrm{s}}} \alpha_{\text {turb }}^{2 q-1}$.

In this above equation $q$ is a parameter characterizing the turbulence. We take $q=1 / 2$ in this paper (Schräpler \& Henning 2004).

With the vertical settling velocity and the turbulent mixing constant we can now define the settling/mixing equation as follows:

$\left.\frac{\partial f(m)}{\partial t}\right|_{\text {sett }}=-\frac{\partial\left(f v_{\text {sett }}\right)}{\partial z}+\frac{\partial}{\partial z}\left(D(z) \rho \frac{\partial(f / \rho)}{\partial z}\right)$.

In this paper we solve this equation for each radius $R$ separately. We do not couple these radii, i.e. particles are not allowed to move from one vertical slice to another. In principle this should be included, since radial drift can be very important. But for simplicity, and since we are mainly interested in the growth from $0.1 \mu \mathrm{m}$ grains to $1 \mathrm{~cm}$ grains, we ignore this process and treat every radius as a separate $1 \mathrm{D}$ vertical settling/mixing problem, coupled to the coagulation equations described below.

We solve the settling/mixing equation numerically using implicit differencing. This is necessary since the CourantFriedrich-Lewy condition for the vertical mixing would require much too small time steps for an explicit solution, and would make the simulation prohibitively slow.

\subsection{Coagulation}

The coagulation of grains is a $1 \mathrm{D}$ time-dependent problem in $m$, which has to be solved at each $R$ and $Z$. The coagulation equation is (Schumann 1940; Todes 1949; Safronov 1969):

$$
\begin{aligned}
\left.\frac{\partial f(m)}{\partial t}\right|_{\text {coag }}= & \int_{0}^{m / 2} f\left(m^{\prime}\right) f\left(m-m^{\prime}\right) \sigma_{\mathrm{c}}\left(m^{\prime}, m-m^{\prime}\right) \\
& \times \Delta v\left(m^{\prime}, m-m^{\prime}\right) \mathrm{d} m^{\prime} \\
& -\int_{0}^{\infty} f\left(m^{\prime}\right) f(m) \sigma_{\mathrm{c}}\left(m^{\prime}, m\right) \\
& \times \Delta v\left(m^{\prime}, m\right) \mathrm{d} m^{\prime}
\end{aligned}
$$


which is the continuous form of the Smoluchowski equation (Smoluchowski 1916). The first term on the right hand side represents the gain of dust matter in the mass bin $m$ by coagulation of two grains of mass $m^{\prime}$ and $m-m^{\prime}$. The second term represents the loss of dust matter in the mass bin $m$ by coagulation of a particle of mass $m$ with a particle of mass $m^{\prime}$. $\Delta v\left(m_{1}, m_{2}\right)$ denotes the average relative velocity between these two particles. This average velocity may consist of random motions but also of systematic drift between particles of different mass. The combination $K\left(m_{1}, m_{2}\right) \equiv \sigma_{\mathrm{c}}\left(m_{1}, m_{2}\right) \Delta v\left(m_{1}, m_{2}\right)$ is called the kernel of the coagulation equation.

We include three processes leading to a $\Delta v\left(m_{1}, m_{2}\right)$ : Brownian motion, differential settling and turbulence. For Brownian motion, the average relative velocity is given by:

$\Delta v_{\mathrm{b}}\left(m_{1}, m_{2}\right)=\sqrt{\frac{8 k T\left(m_{1}+m_{2}\right)}{\pi m_{1} m_{2}}}$.

This represents an average of random velocities, which is highest when both particles have the smallest mass. Therefore, Brownian motion favors collisions in which at least one collision partner has low mass. Once these lowest mass particles get depleted, the next higher mass particles (which are aggregates of the smallest particles) will coagulate etc. Brownian motion will therefore lead to a narrow size distribution which slowly moves to larger sizes. This hierarchical growth procedure leads to aggregates with a fractal structure: so-called cluster-cluster aggregates (CCA) (Meakin 1987).

Differential settling is the process by which large grains, which settle faster than small grains, sweep up the smaller grains on their way to the midplane. This process is very similar to the formation of rain drops in clouds in the Earth's atmosphere ("rain out"). The systematic relative velocity is:

$\Delta v_{\mathrm{s}}\left(m_{1}, m_{2}\right)=\left|v_{\text {sett }}\left(m_{1}\right)-v_{\text {sett }}\left(m_{2}\right)\right|$.

This is clearly zero for particles with equal $\sigma_{\mathrm{g}} / m$ ratios. In the special case of a uniquely defined $\sigma_{\mathrm{g}} / m$ for any given $m$, as we are using in this paper, this means that the relative velocity is zero for equal mass particles, since they both settle at equal speed. The differential settling as a source of collisions works best for particles of very different $\sigma_{\mathrm{g}} / m$ (and therefore mass). Therefore typically the largest mass aggregates will sweep up the smallest particles. This leads to relatively compact particles with porosities typically of the order of $90 \%$. Such aggregates are often called particle-cluster aggregates (PCA).

Turbulence-driven coagulation is a rather complex process. It requires detailed calculations of the statistics of motions of particles (Voelk et al. 1980). Unfortunately, these calculations are too complex to directly build into a coagulation code like the one we present here. Weidenschilling (1984) has derived fitting formulae to the results of Voelk et al., which we implement with slight modifications here. We defer a discussion of these to Appendix A. It should be noted that these formulae introduce a considerable uncertainty into the present coagulation code, since they may depend on details of the turbulence which are not well known. For example, turbulence driven by the magneto-rotational instability (Balbus \& Hawley 1991) may have different properties than the turbulence assumed by
Voelk et al. The result we focus on in the current paper, the fast disappearance of small grains, is present even if we ignore turbulence as a source of relative velocities. Therefore, the uncertainties introduced by assuming a particular turbulence spectrum are acceptable.

Solving Eq. (5) numerically is challenging. In Appendix B we describe our numerical algorithm, and in Appendix $C$ we show the results of a comparison against a test case described in the literature.

\subsection{Grain fragmentation}

In most of this paper we shall ignore aggregate fragmentation/destruction, since we are interested in what happens to the observables of the protoplanetary disk when coagulation is able to develop to its full extent. But in Sect. 6 we will present a single-vertical-slice calculation of a situation in which grain fragmentation is included. Including grain fragmentation in a proper way is challenging, but since the purpose of that section is only to demonstrate the main effects of replenishment of the smaller grains, we are satisfied with an extremely simplified implementation of fragmentation. We assume two colliding aggregates to disintegrate entirely into monomers if their collision energy, when divided over the sum of the masses of the two particles, exceeds a certain value. As our fragmentation energy we choose $1250 \mathrm{erg} / \mathrm{g}$, which, for equal mass particles, amounts to a collision velocity of $1 \mathrm{~m} / \mathrm{s}$. This represents rather loosely bound particles, which enhances the effect of fragmentation. In reality somewhat higher fragmentation velocities may be required, and also other processes such as partial fragmentation (or cratering) may occur. But for demonstration of principle this very simple recipe is justified.

This is implemented in the following way. The mass bins below $a \lesssim 0.5 \mu \mathrm{m}$ are considered to be "monomer bins". Any collision between particles of size larger than $0.5 \mu \mathrm{m}$ with a collision energy (divided by mass) larger than the critical value will remove the mass from the two original bins and return this mass into the monomer bins. The distribution in which it is returned is fixed, and represents the starting distribution shape (MRN, see below).

\section{Prelude: The one-particle model}

Before we present our full-fledged coagulation-settling-mixing models we briefly revisit the simple one-grain model discussed by Safronov in his book (1969), since this model is very illustrative of the phenomena presented in the subsequent sections. The one-particle model follows the growth and settling of a single dust particle in a disk, assuming that all other dust particles remain suspended in the disk and do not coagulate. As the particle settles, it sweeps up the small grains suspended in the disk. Therefore, the mass of the particle is an increasing function of time: $m(t)$ and the height of the particle above the midplane $z(t)$ decreases. Here, and in the remainder of the paper, we take a very simple disk vertical structure in order not to complicate matters more than necessary. We assume that the 
disk is vertically isothermal and the gas density obeys hydrostatic equilibrium. We therefore have:

$\rho(z)=\frac{\Sigma}{\sqrt{2 \pi} H_{\mathrm{p}}} \exp \left(\frac{-z^{2}}{2 H_{\mathrm{p}}^{2}}\right)$,

where $H_{\mathrm{p}}$ is defined as

$H_{\mathrm{p}}=\frac{c_{\mathrm{s}}}{\Omega_{\mathrm{K}}}=\sqrt{\frac{k T}{\mu m_{\mathrm{p}}} \frac{R^{3}}{G M_{*}}}$,

with $M_{*}$ the mass of the central star, $\mu$ the mean molecular weight, taken to be $\mu=2.3$ (for molecular gas) and $R$ the distance from the star. The temperature $T$ is assumed to be the midplane temperature of a disk irradiated under an angle of $\alpha_{\text {irr }}=0.05$ around a star of $M_{*}=0.5 M_{\odot}, T_{*}=4000 \mathrm{~K}$ and $R_{*}=2.5 R_{\odot}$. If we assume an isothermal disk structure without a warm surface layer, then the temperature of such a disk is $T=\alpha_{\text {irr }}^{1 / 4} \sqrt{R_{*} / R} T_{*}=204 \mathrm{~K}$.

Now if we let the particle in question settle toward the midplane according to the settling velocity Eq. (1), then the equation of motion for that particle becomes:

$\frac{\mathrm{d} z}{\mathrm{~d} t}=v_{\text {sett }}=-\frac{3 \Omega_{\mathrm{K}}^{2} z}{4 \rho c_{\mathrm{s}}} \frac{m}{\sigma_{\mathrm{g}}}$.

At the same time, the mass of the particle increases according to

$\frac{\mathrm{d} m}{\mathrm{~d} t}=0.01 \rho(z)\left|v_{\mathrm{sett}}\right| \sigma_{\mathrm{c}}(m)$,

where the factor 0.01 is the dust-to-gas ratio. The above two equations form a coupled set of ordinary differential equations which can be integrated using standard integration schemes. Since the evolving particle quickly is much larger than the particles suspended in the gas, we assume $\sigma_{\mathrm{g}}=\sigma_{\mathrm{c}}$ - but we will see below and in Sect. 4 that this is not fully correct.

In Fig. 1 we show the resulting $z(t), m(t)$ and $a(t)$ assuming spherical compact silicate grains in a disk with $\Sigma_{\text {gas }}=$ $10^{2} \mathrm{~g} / \mathrm{cm}^{2}$ at $R=1 \mathrm{AU}$, starting at a height $z=4 H_{\mathrm{p}}$ (with $H_{\mathrm{p}}$ the pressure scale height of the disk given by $H_{\mathrm{p}}=$ $\sqrt{\left.k T R^{3} / \mu m_{\mathrm{p}} G M_{*}\right)}$, and with grains of different initial radius (mass). As one can see from these figures, the grain grows exponentially as it sweeps up matter during its decent. It reaches the midplane as a cm size pebble in a few hundred years, even though it would have taken a few million years to reach the midplane if the grain would not have grown to larger size on its way to the midplane. Interestingly the time it takes to reach the midplane is almost independent of the initial grain size. In fact, it is also almost independent of the compactness of the grains, as can be seen in Fig. 2. A porous grain has lower settling velocity but larger cross-section. It therefore sweeps up more matter, allowing it to regain the velocity it would have had if it had been more compact (Weidenschilling 1997b). Moreover, the porous grain ends up at the midplane with a larger mass than a compact grain. If one were to redo the experiment with fractal grain growth (resulting in cluster-cluster aggregates) the resulting end size of the grain diverges completely, becoming

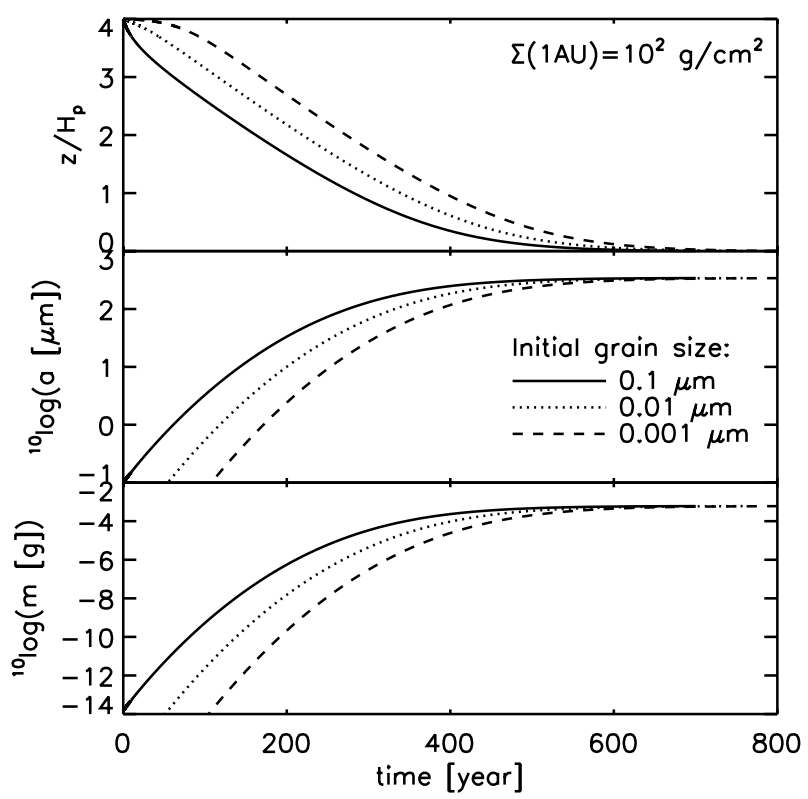

Fig. 1. The time-evolution of the height $z$, radius $a$ and mass $m$ of a dust grain in the simple one-particle model, for three different initial dust radii. The specific weight of the dust is $3.6 \mathrm{~g} / \mathrm{cm}^{3}$.

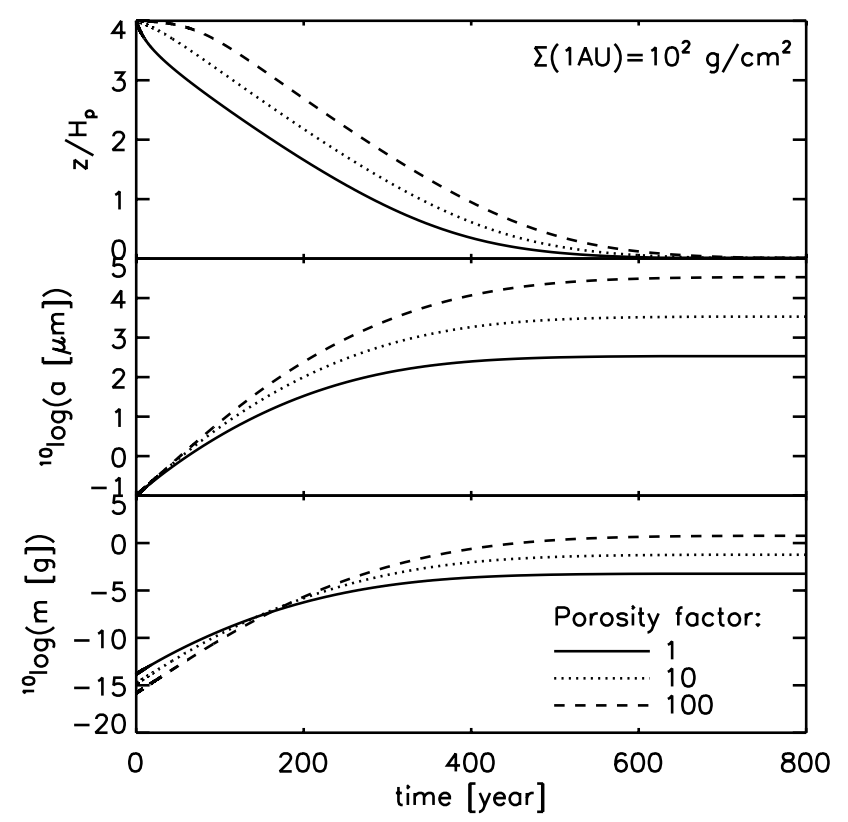

Fig. 2. As Fig. 1, but now for different porosities of the grains.

(theoretically) larger than the entire disk but with virtually infinite porosity. This is clearly unphysical. In reality, at some particle size a transition to more compact particles with fractal dimension 3 will take place, avoiding this divergence. At what size and how this happens is still one of the main questions in the study of planet formation.

As a preliminary conclusion from this simple exercise one can say that grain growth due to this "raining effect" happens on a very short time scale, much shorter than the typical life time of protoplanetary disks. Also it seems that porousness or 
fluffiness of the grain does not slow down this growth process. It merely increases the final grain mass.

\section{Local disk models with coagulation, settling and mixing}

The result of the previous section has shown that coagulation can proceed very quickly through the differential settling process. However, that calculation was based on the simple assumption that a single grain follows this mode of growth while all other grains remain suspended in the gas. In reality this is not the case. All grains evolve simultaneously, and settle simultaneously. It is not clear at what height above the midplane this "rain-out" process starts, and what the effect of the collective growth is. Moreover, as will be shown below, the initial mode of growth is Brownian motion, not differential settling, and only after a certain time will the differential settling take over.

In this section we will show the results of the full-fledged coagulation-settling-mixing calculations following the equations outlined above. We do the calculation first for a single vertical slice at $R=1 \mathrm{AU}$. As our disk vertical structure we adopt the same structure as in Sect. 3. Our initial distribution function is a Mathis et al. (1977) (MRN) distribution from $0.1 \mu \mathrm{m}$ to $0.5 \mu \mathrm{m}$.

In order to show all the effects more clearly, we proceed in steps. First we assume no settling, nor mixing nor coagulation by differential settling nor coagulation by turbulence: we only include Brownian motion (model S1). Then we show a model in which we include the raining effect as well (the coagulation by differential settling), but still no vertical mixing (model S2). We then also include turbulent mixing (model S3). Finally we also include coagulation by turbulence (model S4). These models will demonstrate the speed at which the coagulation takes place.

The models S1, S2, S3 and S4 are for compact spherical silicate grains. In order to show what the effects of non-compactness would be, we also present models similar to S4, but with particle-cluster aggregates (PCA) and cluster-cluster aggregates (CCA). These are the models S5 and S6 respectively.

Table 1 provides an overview of the models of this section. The resulting midplane dust distributions at different times are are shown in Fig. 3. In these plots, we show the actual mass distributions (i.e. $m^{2} f(m)$ when plotted over $\log m$ or $m \cdot a \cdot f(a)$ when plotted over $\log a$, like $v F_{v}$ for spectral energy distributions). On the $\mathrm{x}$-axis we deliberately plot the radius of the grain $a$ instead of the mass $m$, because the radius of grains is a more familiar quantity. For the PCA and CCA models (models S5 and S6) this radius is the equivalent radius as if the grain would have been compact (i.e. an equivalent radius $a$ for the PCA/CCA models corresponds to the same particle mass as for the compact models). For the PCA/CCA models the real radius is much larger.

For clarity, we plot the results in two different ways. Figure 3 shows the midplane size distributions at different times for the various models in a normal 2D plot. Figure 4 shows the same results in a 3D way, i.e. we provide a separate
Table 1. Table of parameters of single vertical slice models (the S-series). First column: model name, second column: Brownian motion, third column: differential settling ("rain effect"), fourth column: turbulent mixing, fifth column: turbulence-driven coagulation and sixth column: porosity (Compact, Particle-Cluster-Aggregate or Cluster-Cluster-Aggregate).

\begin{tabular}{l|ccccc}
\hline \hline & Brownian & DiffSett & TurbMix & TurbCoag & Poros \\
\hline S1 & $\sqrt{ }$ & & & & Comp \\
S2 & $\sqrt{ }$ & $\sqrt{ }$ & & & Comp \\
S3 & $\sqrt{ }$ & $\sqrt{ }$ & $\sqrt{ }$ & & Comp \\
S4 & $\sqrt{ }$ & $\sqrt{ }$ & $\sqrt{ }$ & $\sqrt{ }$ & Comp \\
S5 & $\sqrt{ }$ & $\sqrt{ }$ & & & PCA \\
S6 & $\sqrt{ }$ & $\sqrt{ }$ & & & CCA \\
\hline
\end{tabular}

axis for time. This is useful in particular for the calculations in which the size distribution develops two different peaks - such development gets confusing in the 2D plot. We plot $m \cdot a \cdot f(a)$ as a function of $\log a$ so that surface under the distribution function corresponds to total density of dust at that position in the disk.

As one can see from the upper left panels of Figs. 3 and 4, the coagulation by Brownian motion tends to produce a peak distribution with a certain width. The peak moves toward larger sizes in a self-similar way. But the speed with which it moves toward larger sizes is proportional to $a_{\text {peak }} \propto t^{2 / 5}$. This can be understood in very simple terms. If we assume for simplicity that we have to deal with a mono-disperse size distribution of compact particles with size $m(t) \propto t^{\mu}$, it is clear that the number density of particles is proportional to $n(t) \propto m(t)^{-1} \propto t^{-\mu}$. The relative velocity is $v(t) \propto m(t)^{-1 / 2} \propto t^{-\mu / 2}$. For the compact particles in this calculation, the collisional cross section is $\sigma(t) \propto m(t)^{2 / 3} \propto t^{2 \mu / 3}$. The change in mass of the particles is given by $\mu t^{\mu-1}=\frac{\mathrm{d} m}{\mathrm{~d} t} \propto m(t) n(t) \sigma(t) v(t)$. Comparing the powers to which $t$ is raised in this equation we easily find $\mu-1=\mu-\mu+2 \mu / 3-\mu / 2$ with the solution $\mu=6 / 5$ implying $m(t) \propto t^{6 / 5}$ and $a(t) \propto t^{2 / 5}$. While for the very smallest grains this is an important growth mechanism, clearly this is not efficient for growth to very large sizes.

If the differential settling is included (model S2) the initial stage of growth at the midplane is still Brownian motion. But at some point ( $t \simeq 500$ year) one can see a sudden "rain shower" appearing around grain sizes of $a \simeq 1 \mathrm{~mm}$. This differential settling has already started at higher elevations before that time, but has not yet reached the midplane until $t \simeq 500$ year. The abrupt appearance of $a \simeq 1 \mathrm{~mm}$ size grains at $t \simeq 500$ year means that the "rain drops" have finally reached the midplane and populate the midplane mass bins of size $a \simeq 1 \mathrm{~mm}$, giving rise to the isolated peak distribution around this size seen in the upper-right panel of Fig. 3. During the "rain shower" the height of the original peak of the distribution function (at smaller grain sizes) is strongly reduced: these small grains are swept up by the descending larger particles. This process is similar to what happens in the Earth's atmosphere when aerosols (smog particles) are washed out of the sky by rain. 


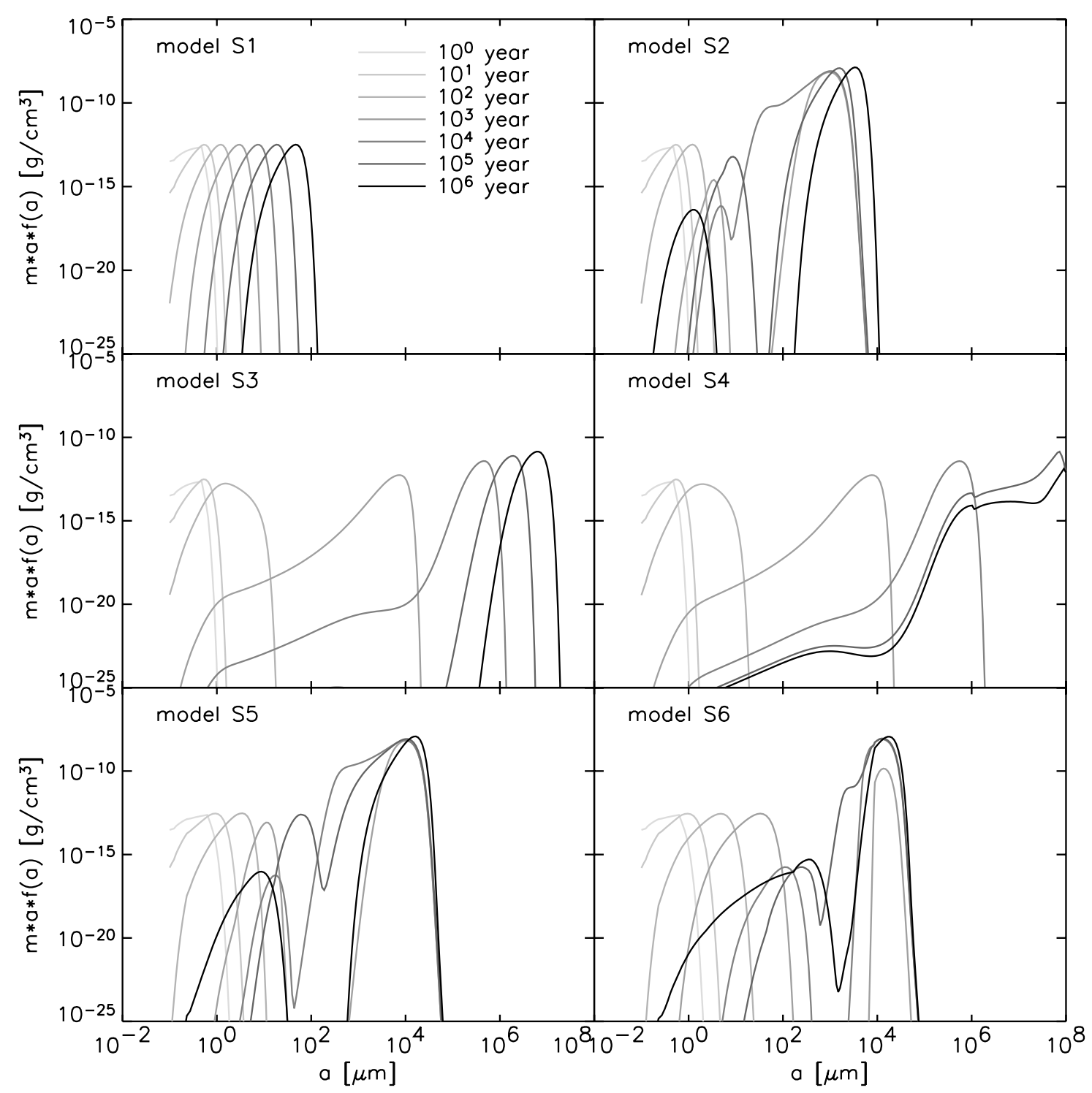

Fig. 3. The time-evolution of the distribution function for models S1 $\cdots$ S6 (see Table 1 for the model definitions).

The time scale for this "rain shower" to reach the midplane is very similar to that predicted by the one-particle model. The particles are, however, about 3 times larger than in the one-particle model. This is because the collective raining allows grains to coagulate with particles of similar (though not equal) size, thereby increasing the collision cross-section of the grains. The maximum increase factor would be a factor of $\sigma_{\mathrm{c}} / \sigma_{\mathrm{g}}=4$, if the grains were allowed to coagulate with equal size partners. But since the differential settling only works for particles of unequal size, this factor is reduced somewhat, i.e. becoming a factor of 3 in our simulation.

It is interesting to see that the peak value of $m \cdot a \cdot f(a)$ of the rained-down grain population (after 500 years) is much higher than the peak value of the initial distribution, even though the peak width is not much different from the width of the original distribution. This may appear as a violation of mass conservation. The explanation is that due to the settling virtually all the dust grains larger than about $10 \mu \mathrm{m}$ have settled in an extremely thin midplane layer with very high dust density.
This is the "dust subdisk" in which, as is sometimes believed, gravitational instabilities may trigger planetesimal formation (Goldreich \& Ward 1973; Youdin \& Shu 2002). This thin midplane layer is allowed to form in model S2 because we have explicitly switched off turbulence. In practice there is doubt whether such a thin layer can exist, as even the slightest bit of turbulence thickens the dust subdisk (Weidenschilling 1995)

After the "rain-out" there is little change, since the "rain shower" has cleared out a large fraction of the small grains in the disk, and there are not enough of them left to continue the raining effect. Basically, the rain shower is over. The remaining population of small grains continues to grow via Brownian motion until it merges in the population of rained-down grains. It should be noted, however, that a minute amount of small grains from higher elevations, which have survived the rain shower, still slowly settle toward the midplane. These grains reach the midplane during the later stages of the simulations. The slightly larger grains arrive first, the smallest grains last. Once arrived at the midplane, the grains are incorporated into larger grains 
model S1
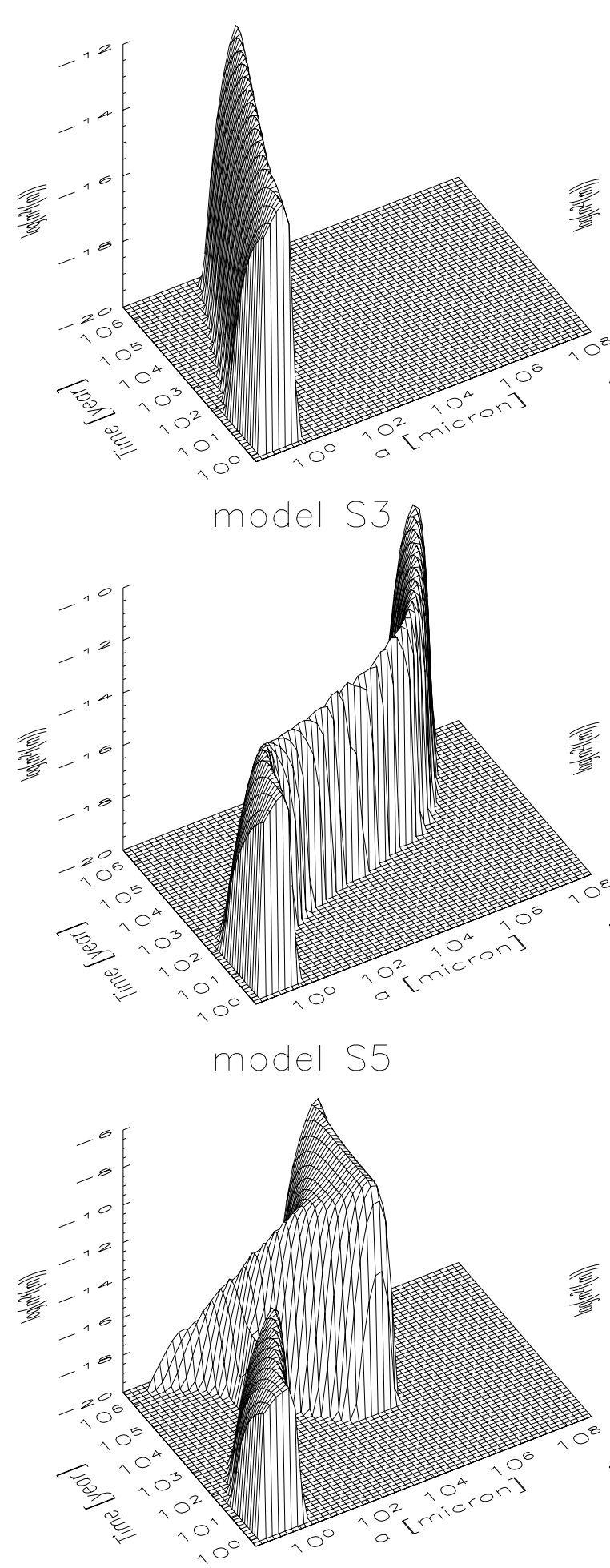

Fig. 4. Like Fig. 3, but in a 2d view.

due to Brownian motion. The combined effect is a dip in the size distribution around about a size of $10 \mu \mathrm{m}$. This dip widens with time, producing a size distribution with two peaks. Due to the arrival of ever smaller grains, the small particle peak moves to the left, while the large particle peak slowly moves to the model S2

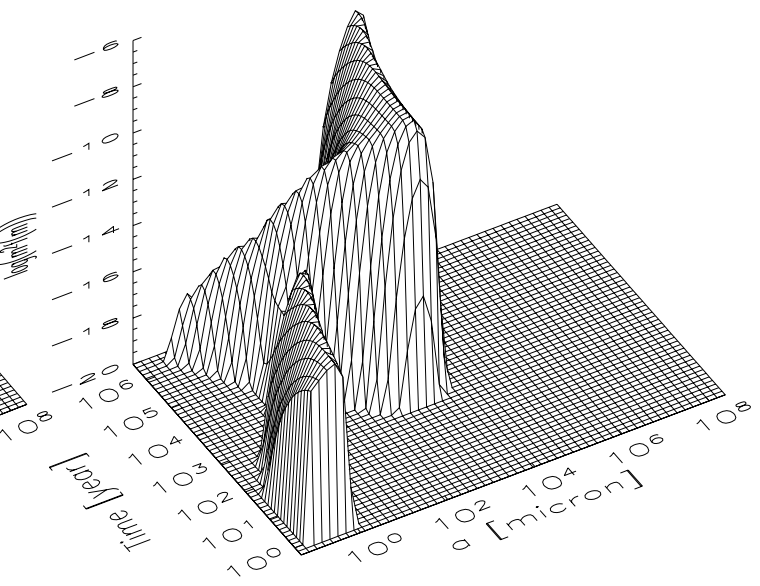

model S4

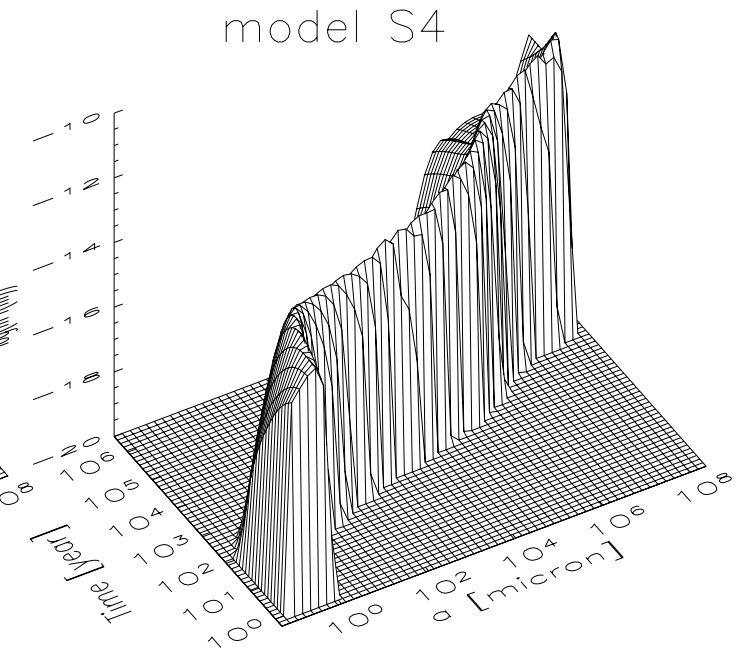

model S6

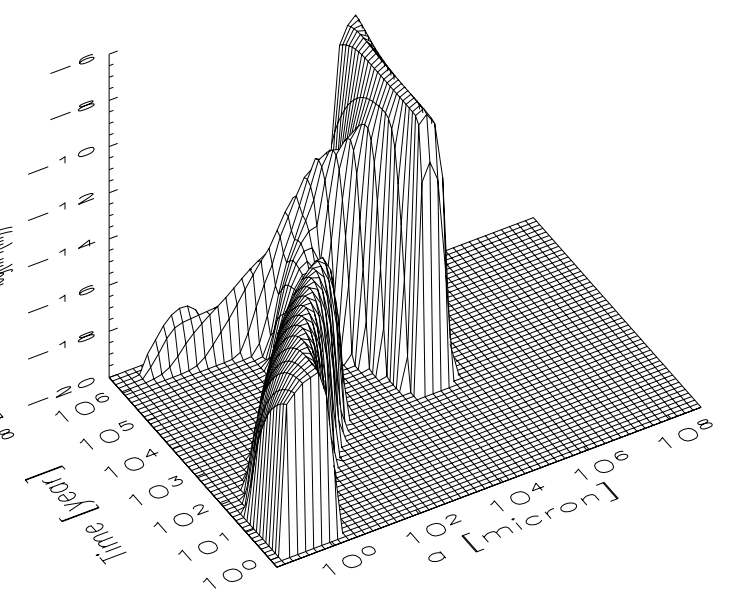

right (because of further Brownian motion grain growth). If the small grains would not be incorporated into the big grains (for instance, if one would switch off Brownian motion coagulation), the settled size distribution would freeze, with the dip between the two peakes filled in. 
Around $1 \mathrm{Myr}$ the secondary peak is seen at $1 \mu \mathrm{m}$. It appears as if this peak is only 10000 times smaller than the value of the initial distribution. While this is a strong depletion of small grains, it may not be enough to render the disk optically thin. However, here again this is somewhat deceptive due to the thinness of the dust subdisk. For grains of this size the dust subdisk is $1 \%$ of the pressure scale height of the disk at $1 \mathrm{Myr}$. This brings the depletion factor to about $10^{6}$.

If we include the vertical turbulent mixing with $\alpha_{\text {turb }}=0.01$ (model S3), then a new interesting phenomenon occurs. The initial evolution remains the same as for S2 (Brownian motion followed by raining), but the differential settling process does not stop anymore. Because the rained-down grains are now stirred up from the midplane again, they can rain down a second and third time, continuing to sweep up any grains they meet. This prolongs the fast growth process considerably, and strongly depletes the small grains from the disk. This process is somewhat similar to the growth of giant hail stones in cumulonimbus clouds in the Earth's atmosphere: before such stones reach the Earth's surface, they often get transported back to high altitudes by the strong updraft within the cloud. In this way these hail stones get multiple chances to collect rain drops and smaller hail stones on their surface, allowing them to grow to sizes as large as decimeters. The main difference is that the air movement in cumulonimbus clouds constitutes a systematic flow, while in our models the turbulent motions are random.

In model $\mathrm{S} 4$ we also include the coagulation by the turbulence itself. This process does not appear to make much difference in the earlier phases of the coagulation process. The differential setting effect (prolonged by the vertical stirring) is clearly the dominant process up to $10^{4}$ years. After that, the turbulence-driven coagulation takes over and manages to grow the grains even further. However, by this time we are well in the regime of boulders, in which many of the equations we use are no longer valid. Note a tiny wiggle in the distribution function at $10^{6} \mu \mathrm{m}$ : this is an effect that can be traced back to the discrete jump in the slope of the fitting formulae of Appendix A.

From the above calculations it is clear that, if we allow coagulation to work with perfect efficiency (i.e. maximum sticking, no destruction) coagulation happens on an extremely short time scale, even if we only include coagulation by Brownian motion and differential settling into our calculations. If we would include other processes such as coagulation by radial drift, while still assuming perfect efficiency, then this is only aggravated instead of alleviated. It seems therefore that this result is rather robust. We shall see in Sect. 6 that destructive collisions may indeed play an important role.

\subsection{The optical depth of the models}

A first hint of the effects of the coagulation on the appearance of a disk can be taken from the optical depth at short wavelength, i.e. at wavelength corresponding to the stellar photons which are heating the disk surface and are responsible for the flaring in the disk.

In Fig. 5 we show the vertical optical depth at UV wavelengths through the disk. For the cross section we have taken

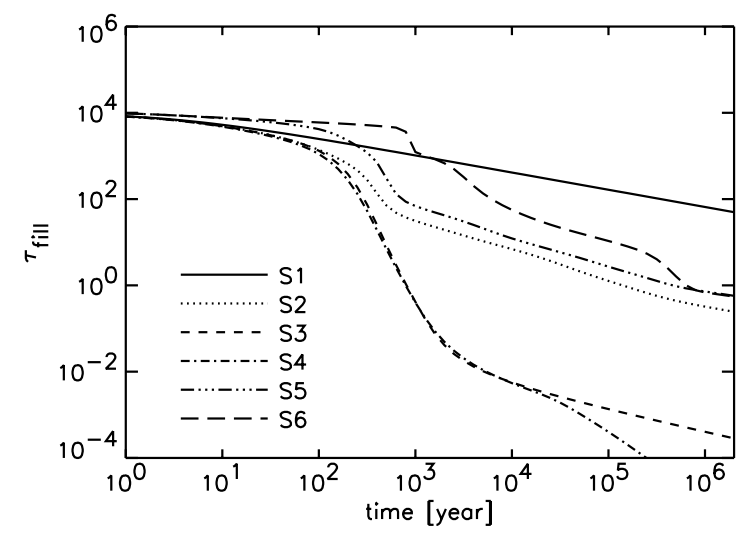

Fig. 5. The vertical optical depth in the UV at 1 AU (i.e. the integrated projected surface of the dust particles) as a function of time for the vertical slice models $\mathrm{S} 1 \ldots \mathrm{S} 6$.

the projected geometrical cross section, i.e. $\sigma_{\mathrm{g}}$. We can see that for all models, the optical depth decreases significantly due to the coagulation. While in the pure Brownian Motion case S1, the optical depth decreases by a factor of 100 in $10^{6} \mathrm{yrs}$, including settling already decreases the optical depth by more than four orders of magnitude in the same time, pushing the optical depth below 1 . With turbulent mixing and turbulent coagulation, the effect becomes enormous, and the disk becomes fully transparent in only $1000 \mathrm{yrs}$. Using PCA and CCA grains does slow down the process somewhat. In particular, in the CCA calculation the optical depth stays constant for about 1000 years, but then also starts to decrease quickly. After $10^{6}$ years, also in this case the optical depth is below one. The possibility of change in optical depth on short time scales was also indicated by Weidenschilling $(1980,1997 a)$ who found that the Rosseland optical depth may decrease by an order of magnitude in about 1000 orbital periods.

\section{Global disk models with coagulation, settling and mixing}

The short coagulation time scales will have consequences for the infrared and optical appearance of protoplanetary disks. In this section we combine a series of vertical slices (annuli) to form a full disk model. We perform the coagulation-settlingmixing calculations in each of these slices and take snapshots of the distribution function at given times. From this series of slices we construct a 3D axisymmetric (i.e. effectively 2D) disk model at each of the snapshot times, and feed these disk models into a multi-dimensional continuum radiative transfer code called RADMC (see Dullemond \& Dominik 2004a). With this code we can produce synthetic spectral energy distributions (SEDs) for the disk at the given times, as well as images at various wavelengths and inclinations.

Our model has an inner radius $R_{\text {in }}=0.7 \mathrm{AU}$, outer radius $R_{\text {out }}=100 \mathrm{AU}$ and has a gas surface density profile $\Sigma_{\text {gas }}(R)=\Sigma_{0}(R / \mathrm{AU})^{p}$ with $p=-1.5$ and $\Sigma_{0}=400 \mathrm{~g} / \mathrm{cm}^{2}$, which amounts to a disk with $M_{\text {disk }}=0.005 M_{\odot}$. In total we combine 40 vertical slices to form the full disk model. The 
Table 2. Same as Table 1, but now for the full disk models F1 and F2.

\begin{tabular}{c|ccccc}
\hline \hline & Brownian & DiffSett & TurbMix & TurbCoag & Poros \\
\hline F1 & $\sqrt{ }$ & $\sqrt{ }$ & & & Comp \\
F2 & $\sqrt{ }$ & $\sqrt{ }$ & $\sqrt{ }$ & & Comp \\
\hline
\end{tabular}

radii of these slices are $\log$-spaced between $R_{\text {in }}$ and $R_{\text {out }}$, meaning that the width of each slice is about $14 \%$ of its radius. We take the same stellar parameters as in previous sections, and similarly we assume compact silicate grains. The temperature of the disk is a function of radius $R$ only, i.e. it is constant in the vertical direction. As in the previous sections, the temperature is assumed to follow from thermal balance assuming that the disk is passive and irradiated by the star at an angle $\alpha_{\text {irr }}=0.05$, leading to $T(R)=\alpha_{\text {irr }}^{1 / 4} \sqrt{R_{*} / R} T_{*}$. We present two models. Model F1 has no turbulent stirring (we set $\alpha_{\text {turb }}=0$ ) while model F2 has turbulent stirring with $\alpha_{\text {turb }}=0.01$ (see Table 2).

The left column of Fig. 6 shows the evolution of the SED of the full disk model without turbulence (F1). One sees that very early the mid-IR drops, while the far-IR remains. This is because the coagulation processes are faster at small radii, and therefore the depletion of small grains happens faster at small radii than in the outer regions of the disk. It is also clear that the $10 \mu \mathrm{m}$ feature quickly loses strength but does not disappear before most of the mid-IR continuum flux disappears as well. Its shape also does not change appreciably. This is an interesting phenomenon, since observations of the $10 \mu \mathrm{m}$ feature of $\mathrm{T}$ Tauri stars and Herbig Ae/Be stars often show flattening and weakening of this feature which is generally interpreted as a signature of grain growth (van Boekel et al. 2003; Przygodda et al. 2003; Meeus et al. 2003; Honda et al. 2003). According to the present calculations such flattened features are not predicted. Moreover, the entire mid-IR flux vanishes much too quickly (well within $10^{5}$ years). In fact, by $10^{6}$ years most of the IR excess (also far-IR) has vanished, which is clearly inconsistent with observations of $\mathrm{T}$ Tauri stars and Herbig Ae/Be stars. The same behavior is reflected by the surface height in the disk, plotted in the upper right panel of Fig. 6. The surface height is calculated by following starlight radially away from the stellar surface and determining at what location an optical depth of unity for a wavelength of $0.55 \mu \mathrm{m}$ is reached. In contrast to the vertical optical depth shown for the slab calculations (see Fig. 5), this plot is also sensitive to the vertical distribution of the material. It measures both loss in total optical depth, and settling of the opacity carriers toward the midplane and therefore is the most important indicator for understanding the SED. The surface height initially begins to decrease globally, which is mainly due to the settling motion of particles in the uppermost layers (Dullemond \& Dominik 2004b). In the inner disk regions, the effects of coagulation quickly lead to a transparent disk, with the disk "surface" disappearing. The boundary where photons are intercepted moves outward and reaches $30 \mathrm{AU}$ in $10^{6}$ years, while in the outer disk, the surface height continuously decreases. If one includes turbulence in the model, then the SEDs become as shown in the two bottom panels of Fig. 6. In this case the problem is even more acute.

It is interesting to see that as the mid-IR flux vanishes, the far IR flux temporarily increases. This is because of energy conservation. The dust coagulation happens much faster at small radii than at large radii. As the inner regions of the disk become optically thin, the outer regions are still optically thick and reprocess all the radiation that was previously reprocessed by both the inner and the outer regions of the disk. Once also the outer regions get deprived of their small grains, they too become optically thin and the entire IR excess drops down. Note that the far-IR excess for model F2 (with turbulence) is usually larger than that of model F1. This is because even though the grains grow faster in model F2, they also get swept up higher above the midplane so that the disk can reprocess a larger fraction of the stellar radiation.

The results of this section clearly show that the quick disappearance of the small grains due to the efficient interaction between the differential settling and the turbulent mixing is clearly in contradiction with observations. The absence of turbulence may leave a minute population of small grains, but is not very efficient in solving the problem fully. In the next section we discuss what we believe is the most likely solution to this apparent contradiction with observations. In Sect. 7 we discuss various other possible solutions.

\section{A simple local model including aggregate fragmentation}

So far we have taken a perfect sticking condition: if two grains/aggregates collide, they stick and form a larger aggregate. However, for high collision velocities this is no longer true. In this section we show what happens when aggregates are allowed to disintegrate upon collision. This replenishes the small grain size bins, and may go some way toward solving the time-scale problem. Unfortunately the computational demand for such a calculation is orders of magnitude higher than for a pure coagulation calculation. Therefore we only show the results for a single vertical slice (model SD1).

We include aggregate fragmentation in the very simplified way described in Sect. 2.4: if the collision energy exceeds a certain limit, both grains are destroyed upon impact. We take as our model parameters again the same parameters as in model S4 of Sect. 4.

From Fig. 7 we see that, while the initial stages of growth are identical to those of model S4, very quickly the replenishment of small grains due to fragmentation starts to take place. After about $10^{4}$ years a semi-stationary state is reached for sizes below $a \lesssim 1 \mathrm{~cm}$. This is an equilibrium between grain growth and grain fragmentation. As grain aggregates reach sizes of about $1 \mathrm{~cm}$, most of the aggregates are destroyed and their mass returned to monomers, where it immediately starts to coagulate again. For grain sizes larger than $1 \mathrm{~cm}$ the distribution function continues to evolve, forming a powerlaw "tail" with a growing peak value. This is a tail distribution of "lucky" grains which managed to avoid an encounter with an equal size collision partner and therefore avoided fragmentation. These grains sweep up some of the 

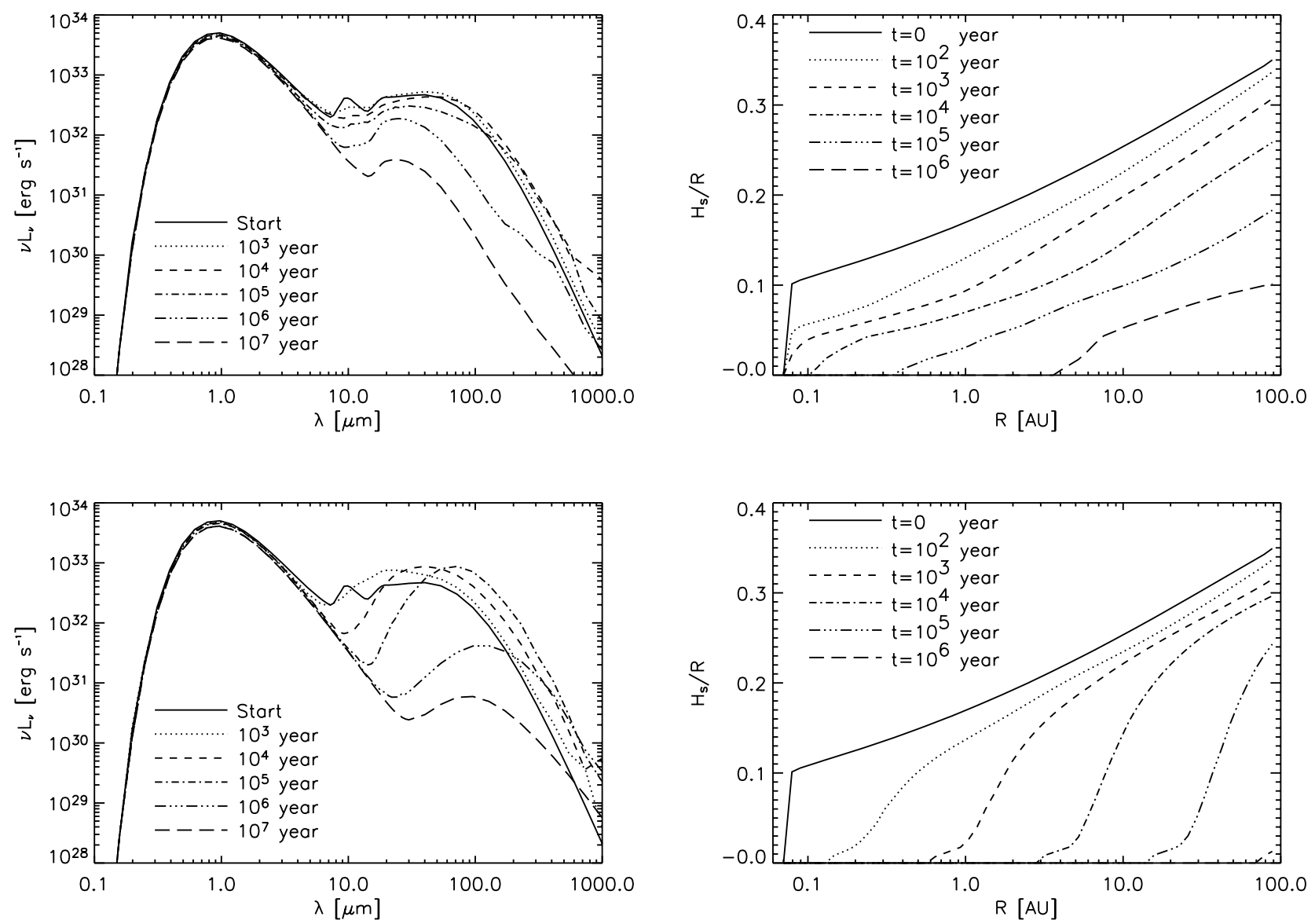

Fig. 6. The SEDs (left column) and surface heights (right column) of the full disk models. The two top panels show the result for model F1, the calculation without turbulence. The bottom panel results are for model F2, including turbulence. Different lines indicate the results after different times, as shown in the legends.

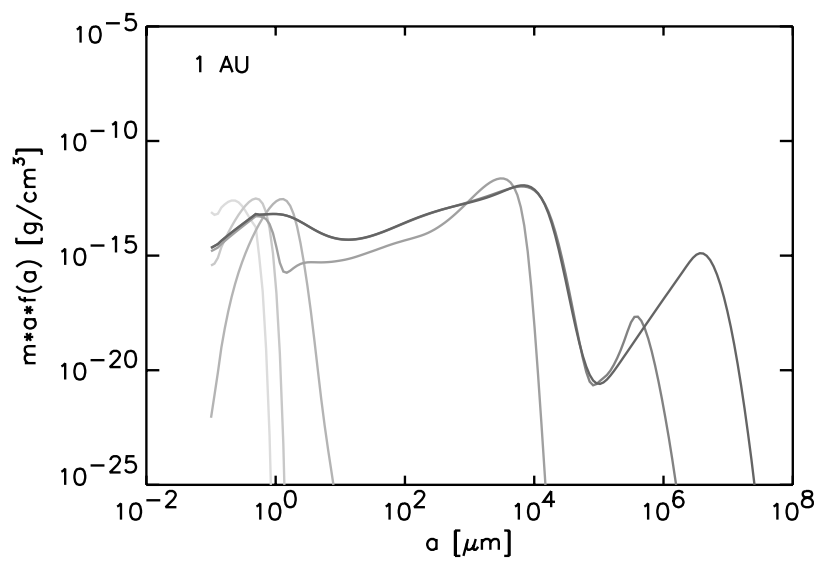

Fig. 7. The time-evolution of the distribution function for model SD1 (including all growth processes and aggregate fragmentation). This slice has only been evolved up to $8 \times 10^{5}$ years (darkest curve).

small grains from the semi-stationary distribution below $1 \mathrm{~cm}$, and therefore manage to grow toward larger sizes. By this time other important effects, which we have not taken into account yet, will become dominant, such as radial drift and the consequent run-away growth. Also some of our equations start to become invalid for such large particles, in particular our formula for the gas drag in the Epstein regime.

\section{Discussion}

\subsection{Other mechanisms to maintain the small particle population}

The basic fact that coagulation will lead to a reduced optical depth in disks has been noted earlier. Weidenschilling (1984) noted that the reduction in opacity may lead to a termination of turbulent gas motions if those motions are driven by convection. Mizuno et al. (1988) forced a steady-state solution for their coagulation calculations by assuming that the disk is constantly supplied with new gas containing new small particles. They also discussed that the addition of new particles may lead to alternating phases of high and low optical depth, and consequently of convection-driven turbulence. However, while turbulence driven by convection may be present in disks, other drivers for turbulence may be important as well. The magnetorotational instability (Balbus \& Hawley 1991) seems to be an important candidate. For the presence of this instability, low gas column densities are required in order to allow cosmic rays 
and $\mathrm{X}$ rays to penetrate and ionize the gas. High gas columns can create so-called dead-zones (Gammie 1996) in the disk mid-plane where ionizing radiation (such as $\mathrm{X}$-rays and cosmic rays) cannot penetrate. Furthermore, the idea of a constant inflow of small dust grains onto the disk can only be valid for the early evolutionary phases of a disk. Observations show that circumstellar disks at an age of several million years still can be strongly flaring (Leinert et al. 2004). By this time, the parental cloud has been largely cleared away and infall of new material as well as accretion onto the star has virtually ceased. The exact limit on the amount of infalling material which can be relevant for the disk opacity is not easily estimated. It will depend on the speed at which vertical mixing and turbulence can remove these grains from the disk. Clearly, for newly added material, the removal time scale will be longer since the low dust density slows down coagulation. More detailed calculations of these effects will have to show if they can resolve the discrepancy between the speed and effectiveness of coagulation on the one hand, and infrared observations of disks on the other hand.

\subsection{The effect of the large grain population on the small grains}

The choice of upper boundary to the grain size $\left(a_{\max }\right)$ in the above simulations has a strong influence on the results for the case in which both differential settling and turbulent mixing are included in the calculation. If $a_{\max }$ is taken too small (for instance $1 \mathrm{~mm}$ only), the grain growth is artificially stopped at that size. This means that a comparatively large population of grains remains at a size around $a_{\max }$ (while it should have grown further), providing a comparatively large cross-section for the depletion of the smaller $(1 \mu \mathrm{m})$ grains. Therefore, choosing $a_{\max }$ to be $1 \mathrm{~mm}$ instead of $10 \mathrm{~m}$ or more strongly enhances the depletion of the small grains. In our simulations we therefore had to choose $a_{\max }$ to be very large (we took it $100 \mathrm{~m}$ ). But this introduces yet another problem: our equations are no longer accurate at those large sizes. Moreover, large bodies tend to drift inward toward the star at a very high pace. This is not included in the present model, because each vertical slice is assumed to be independent of the other slices. Future investigations will have to deal with this problem. However, it is not likely that that would solve the problem of the quick depletion of small grains, since even for the non-turbulent case (which represents the slowest growth) the depletion is quite strong.

\subsection{The inner regions of the disk}

Close to the central star (the inner regions of the disk) the depletion of small grains goes the quickest, since the Kepler time scale is the shortest there. The problem of the quick depletion of small grains seems therefore to be most acute in those regions. But can we be sure that small grains indeed exist so close to the star? The answer seems to come from very recent observations with the mid infrared interferometer MIDI on the Very Large Telescope. Using this instrument, van Boekel et al. (submitted) separated the correlated $8-13 \mu \mathrm{m}$ flux from the total 8-13 $\mu \mathrm{m}$ spectrum for $3 \mathrm{Herbig} \mathrm{Ae} / \mathrm{Be}$ stars. In this way they were able to single out the spectrum from the inner $2 \mathrm{AU}$ region of the disk. Although the $10 \mu \mathrm{m}$ silicate feature in this correlated flux spectrum clearly showed evidence for grain growth up to $2 \mu \mathrm{m}$, it also clearly showed that grains of approximately $2 \mu \mathrm{m}$ were still present and their abundance is strong enough to produce a clearly discernible $10 \mu \mathrm{m}$ feature. This is in clear contradiction with the pure-coagulation models presented here, and reinforce our conclusion that aggregate fragmentation (or some other resupply of small grains) should play a major role in disks.

\subsection{Interpretation of the results}

The models of grain growth presented in this paper show that coagulation happens on a time scale that is two to three orders of magnitude too short to be consistent with observations of T Tauri star disks or the disks around Herbig Ae/Be stars. Turbulent mixing combined with coagulation through differential settling is highly efficient at removing the small grains from the disk at all heights above the midplane. One may argue that some disks may have regions of zero turbulence (for instance the "dead zone" introduced by Gammie 1996), which may decrease the efficiency of the grain growth. But even if we only have differential settling, but no vertical mixing (as in model S2) then only a tiny population of small grains remains in the disk, containing less than $10^{-6}$ of the original population of small grains. The full disk model F1, which is without turbulence, clearly shows that at typical ages of T Tauri stars (around $1 \mathrm{Myr}$ ), almost no IR emission is left, and for the model F2 (with turbulence) this is even more dramatic. If anything, the SED looks like that of a debris disk instead of a $\mathrm{T}$ Tauri star disk. But even if we compare the SED at $10^{4}$ years to observed SEDs, we find that the dip in the SED at near- to mid-IR wavelength is rather untypical for most T Tauri stars.

There are, however, a few examples of objects which show conspicuous near/mid-IR dips. A strong near/mid-IR dip in the SED of TW Hydra was interpreted by Calvet et al. (2002) as a signature of a planet which has cleared out the inner disk. The synthetic SED of model F2 at $10^{4}$ years, however, shows a similar dip, and therefore dust coagulation could be an alternative explanation. HD 100546 is a Herbig star which also features a conspicuously weak near-IR excess, which was attributed to a huge gap in the disk (Bouwman et al. 2003). Here again, dust coagulation may be an alternative explanation.

From the results presented in this paper it seems unavoidable that some form of replenishment of small grains is needed to make the model calculations comply with the observations. The only other possibility is that the sticking probability is enormously reduced by some process. Since we are not aware of a process capable of reducing the sticking probability by such a dramatic amount, we believe that replenishment is the only solution. Replenishment by destructive collisions seems to be the most natural way to prevent the small grains from disappearing entirely. In this paper we demonstrated that this could work if we assume very low binding energies of the grains. The process of cratering (a small particle impacting on a bigger one and creating a certain amount of impact debris) may be a 
better way to produce small grains, but we defer a more detailed implementation of aggregate fragmentation to a future paper.

\section{Conclusions}

In this paper we have modeled dust coagulation in protoplanetary disks and computed the SEDs of such disks. We qualitatively compare these SEDs to what is typically observed from $\mathrm{T}$ Tauri star and Herbig Ae/Be star disks, and we conclude that if coagulation is allowed to proceed unhindered (i.e. without fragmentation of aggregates), then the small grains are depleted on a time scale that is three orders of magnitude too short to be consistent with these observations. We have included three coagulation mechanisms in this model (Brownian motion, differential settling and turbulence). The inclusion of only the first two, well understood, processes already shows that the strong and rapid depletion of small grains is unavoidable unless small grains are somehow replenished. The inclusion of a little bit of turbulent mixing will only aggravate matters. It is very difficult to slow this process down, even by grain charging or other mechanisms. Either the grain sticking efficiency is many orders of magnitude less than currently assumed, or the small grain population must be replenished in some way. We suggest that aggregate fragmentation could be such a mechanism. We present a highly simplified model for this and show that a semi-stationary equilibrium sets in in which coagulation and fragmentation are balanced for an extended amount of time. Whether this is the solution to the paradox remains unclear and requires much more detailed simulations.

Acknowledgements. We wish to thank the referee S. Weidenschilling for a fast and insightful report which helped to improve the paper.

\section{Appendix A: Turbulence-driven coagulation}

Turbulent motion in the gas can cause collisions between dust particles. The basic reason for this is that particles with different $\sigma_{\mathrm{g}} / m$ ratio couple to eddies of different size and therefore acquire random velocities. To calculate the motion caused by turbulence, it is necessary to integrate over the contributions of all eddies from the largest scales down to the smallest scales which are set by the condition that the turbulent Renolds number Re equals 1 .

Calculating the gas-dust interaction is complex and has been covered by previous authors (e.g. Weidenschilling 1977; Cuzzi et al. 1993). Here we only describe the basic recipe implemented in our code.

The main particle property that enters the calculation is the stopping time of of a particle which is given by

$t_{i}=\frac{3}{4} \frac{m}{\sigma_{\mathrm{g}}} \frac{1}{\rho c_{\mathrm{s}}}$

which physically is the time in which a particle reacts to changes in the motion of the surrounding gas. Specifically, for a given turbulent eddy, this time scale indicates if the particle will follow the eddy motion or not.
In order to derive the average relative velocities resulting from this mechanism, the full structure and spectrum of the turbulence must be known. Turbulence is often characterized by the velocity and time scale of the largest eddies (see Dullemond \& Dominik 2004b)

$v_{\text {edd }}^{0}=\alpha_{\text {turb }}^{q} c_{\mathrm{s}}$
$t_{\text {edd }}^{0}=\frac{2 \pi}{\Omega_{\mathrm{K}}}$

where $q$ is a turbulence parameter between 0 and 1 . Following Schraepler and Henning (2004) we take it to be $q=1 / 2$. The energy then cascades down to small sizes, until the flow becomes laminar at a turbulent Reynolds number $\mathrm{Re}=1$ in a gas with viscosity

$v_{\text {edd }}^{\mathrm{s}}=v_{\text {edd }}^{0} \operatorname{Re}_{0}^{-1 / 4}$

$t_{\text {edd }}^{\mathrm{s}}=t_{\text {edd }}^{0} \operatorname{Re}_{0}^{-1 / 2}$

with

$\operatorname{Re}_{0}=\frac{\rho v_{\text {edd }}^{0} \lambda_{\text {edd }}^{0}}{\eta} \approx \frac{\rho\left(v_{\text {edd }}^{0}\right)^{2} t_{\text {edd }}^{0}}{\eta}$.

The random motions of the particles must then be calculated by integrating over the contributions of the different eddy scales. For a Kolmogorov spectrum of the turbulence, this has been done numerically by Voelk et al. (1980) and Mizuno et al. (1988). Weidenschilling (1984) has fitted the numerical results with a simple analytical formula, which has also been used for example by Suttner \& Yorke (2001). We will also adopt it, but with two modifications:

1. Because of the lower cutoff to the eddy size spectrum, no random velocities relative to the gas are introduced by turbulence for particles with a stopping time below the turnover time of the smallest eddy, $t_{\text {edd }}^{\mathrm{s}}$. This has been noted by Weidenschilling (1984) and discussed in detail by Mizuno et al. (1988). We use the limiting case provided by the latter authors.

2. The analytical fit produces an overshoot in the limit $t_{2} \rightarrow$ $t_{\text {edd }}^{0}$. If $t_{1} \ll t_{2}$, the analytical fit given by Weidenschilling (1984) and also used by Suttner \& Yorke (2001) leads to $v_{\text {edd }}^{0}=3 v_{\text {edd }}^{0}$ while the numerical results by Voelk et al. (1980) only exceed $v_{\text {edd }}^{0}$ by a few percent. In order to avoid effects cause by this incorrect high collision speeds, we therefore limit the relative velocity caused by turbulence to $v_{\text {edd }}^{0}$.

With both modifications, the recipe for the turbulent collision velocities becomes

$$
\Delta v_{\text {edd }} \begin{cases}\frac{v_{\text {edd }}^{\mathrm{s}}}{t_{\text {edd }}^{\mathrm{s}}}\left|t_{1}-t_{2}\right| \sqrt{\frac{\ln \operatorname{Re}}{2 \sqrt{\operatorname{Re}}} \frac{t_{\text {edd }}^{0}}{t_{1}+t_{2}}} & : \text { if } t_{1}, t_{2} \leq t_{\text {edd }}^{\mathrm{s}} \\ v_{\text {edd }}^{0} & : \text { if } t_{1} \leq t_{\text {edd }}^{0} \leq t_{2} \\ v_{\text {edd }}^{0} \frac{t_{\text {edd }}^{0}\left(t_{1}+t_{2}\right)}{2 t_{1} t_{2}} & : \text { if } t_{\text {edd }}^{0} \leq t_{1}, t_{2} \\ v_{\text {edd }}^{0} \min \left\{1, \frac{3 t_{2}}{t_{1}+t_{2}} \sqrt{\left.\frac{t_{2}}{t_{\text {edd }}^{0}}\right\}}\right. & : \text { otherwise. }\end{cases}
$$




\section{Appendix B: Numerically solving the coagulation equation}

The numerical solution of Eq. (5) is a subtle matter. Consider a discretized grain mass grid $m_{i}$ with $i \in[1, N]$. The first integral on the right hand side can be converted into a sum over $m^{\prime}=m_{k}$ (for fixed $m=m_{i}$ ) with $k=1 \ldots l$ where $l$ is the highest index for which $m_{l} \leq m_{i}$. Unfortunately the value of $m_{i}-m_{k}$ lies generally not exactly at a discrete mass point and therefore the value of $f\left(m-m^{\prime}\right) \equiv f\left(m_{i}-m_{k}\right)$ must be obtained by interpolation. Since $f$ can vary extremely strongly, the interpolation is best done in $\log (f)$ instead of in $f$. Numerical practice has shown that two-point interpolation makes the algorithm more stable than four-point interpolation. The last point in the integral (i.e. the numerical sum) is located at $m / 2$, and in that case $m^{\prime}=m-m^{\prime}$, i.e. both $m^{\prime}$ and $m-m^{\prime}$ are located in between two mass points and similarly an interpolation needs to be done. The integral in the second term on the right hand side of Eq. (5) is easier, since no interpolation is needed.

\section{B.1. Renormalization}

The right-hand-side of Eq. (5) consists of a gain and a loss term. The gain term describes how much matter enters a certain mass bin through coagulation of smaller particles, while the loss term describes how much matter leaves the mass bin through coagulation of this matter with particles of any other size. Typically these two terms are very large numbers which cancel each other almost entirely, except for a tiny amount. It is this tiny amount that is the crucial source term for the coagulation equation. This near cancellation happens then when the gain of matter in a bin is dominated by coagulation between large and small particles. The reason for this near cancellation is best described with an example. If a rock of $1 \mathrm{~kg}$ that hits a dust particle of 1 micron size, formally the rock increases in mass (albeit by an extremely small amount). The rock is therefore removed from its mass bin and put into the mass bin a few picogram toward larger mass. Since the $1 \mathrm{~kg}$ rock may collide with trillions of 1 micron size particles, the gain and loss terms in the $\mathrm{kg}$ mass bin are huge, but virtually identical. The minuscule difference between these two terms determines the eventual growth from $1 \mathrm{~kg}$ to $2 \mathrm{~kg}$ after the rock collects $1 \mathrm{~kg}$ worth of micron size particles.

Numerically this poses a significant challenge. If one simply computes the gain and loss terms, the near cancellation goes astray once the cancellation happens beyond the 14th digit. In effect the near cancellation turns into a perfect cancellation, which is incorrect. To solve the problem the integrals of the gain and loss terms have to be calculated simultaneously. The integrands of both integrals are calculated at the same time, and then subtracted and collected into a single integral. At each $m^{\prime}$ it is checked if the two terms tend to produce a near cancellation. If so, their difference is recomputed using a renormalization technique:

$$
\begin{aligned}
& f\left(m^{\prime}\right) f\left(m-m^{\prime}\right) \sigma_{\mathrm{c}}\left(m^{\prime}, m-m^{\prime}\right) \Delta v\left(m^{\prime}, m-m^{\prime}\right) \\
& -f\left(m^{\prime}\right) f(m) \sigma_{\mathrm{c}}\left(m^{\prime}, m\right) \Delta v\left(m^{\prime}, m\right) \mathrm{d} m^{\prime} \\
& \simeq-f\left(m^{\prime}\right)\left(m-m^{\prime}\right) \frac{\mathrm{d}\left(f\left(m^{\prime \prime}\right) \sigma_{\mathrm{c}}\left(m^{\prime}, m^{\prime \prime}\right) \Delta v\left(m^{\prime}, m^{\prime \prime}\right)\right)}{\mathrm{d} m^{\prime \prime}}
\end{aligned}
$$

which uses l'Hopital's rule. This renormalized version of the integrand is valid only when a near-cancellation takes place, and remains numerically well-determined even for extreme cases of near-cancellation.

\section{B.2. Mass conservation}

By performing the integration in the above described way, total dust mass is not necessarily perfectly conserved. Small errors can increase or decrease the total mass, and since these errors are generally systematic, the risk is high that the dust mass will unlimitedly grow or diminish as the simulation progresses. In our code this is avoided by making small corrections. Denote $S(m)$ as the right-hand-side of Eq. (5). Define now the following two functions:

$$
\begin{aligned}
& S_{+}(m)= \begin{cases}S(m) & \text { where } S(m)>0 \\
0 & \text { where } S(m) \leq 0\end{cases} \\
& S_{-}(m)= \begin{cases}0 & \text { where } S(m)>0 \\
S(m) & \text { where } S(m) \leq 0 .\end{cases}
\end{aligned}
$$

The new right-hand-side $S_{\text {new }}(m)$ is now:

$S_{\text {new }}(m)=S_{-}(m)+\chi S_{+}(m)$

where $\chi$ is defined as:

$\chi=-\frac{\int m S_{-}(m) \mathrm{d} m}{\int m S_{+}(m) \mathrm{d} m}$.

This is generally a tiny correction, and it guarantees mass conservation and thereby prevents unphysical build-up or loss of matter.

\section{B.3. Time step determination for the coagulation}

Since the evolution of the grain size distribution proceeds each time step by adding a source term with the results of the integrals, the magnitude of this time step is set by:

$\Delta t=\xi \min \left(\frac{f(m)}{|S(m)|}\right)$

where $0<\xi \leq 1$ is an accuracy parameter which we usually set to 0.3 in our simulations. Typically this time step is shortest at the smallest $m$, so that the evolution at small $m$ limits the time step of the entire simulation. This could in principle be prohibitively short compared to the total time we wish to evolve our simulation $\left(10^{7}\right.$ years). Fortunately, as the aggregates grow, the smallest mass bins are quickly depleted and can be set to zero once the value of $f(m)$ drops below some floor value. The time step then does not need to be limited anymore by these small mass bins. In practice this means that as the time progresses, the time step becomes larger and $10^{7}$ years can be reached without many problems.

Sometimes, the simulation may get temporarily stuck at relatively small time steps, despite the above mentioned time stepping method. This is caused by the operator splitting between the settling/mixing and the coagulation. While the coagulation equation may attempt to empty a certain mass bin, the 
settling and mixing may fill it up again. This typically happens at the midplane, where settling and vertical mixing may replenish the mass in a certain mass bin by transporting it down to the midplane from higher altitudes. Since the settling and mixing are numerically simulated in an implicit way, allowing the time step to exceed the Courant condition for for settling and mixing by many orders of magnitude, this can cause the instant refilling of the mass bin after the coagulation equation has tried to deplete it. In practice, however, the simulation never gets entirely stuck, and at some point manages again to increase the time step to large enough values to allow the simulation to end in about $20 \mathrm{~min}$ per vertical slice on a Pentium 4 processor. Therefore it is manageable to simulate an entire disk consisting of 20 vertical slices in about $8 \mathrm{~h}$ CPU time.

However, once the aggregate fragmentation is included, the small grains remain present, and the time step remains limited by the smallest mass bin. The above time stepping method then does not work anymore, and the simulation may take large amounts of CPU time even for a single vertical slice. A fully implicit treatment of the coagulation/fragmentation may then be necessary to prevent excessive computational costs.

\section{Appendix C: Test cases}

It is not straightforward to test the numerical algorithm described here with the physics we include into the coagulation equation, since no analytic solutions exist for the kernel we use. Lai et al. (1972) have presented analytical solutions to the coagulation equation with a Brownian motion kernel, but these solutions are only valid in limiting cases and they contain undetermined constants.

For simplified kernels, various analytic solutions to the coagulation equation exist. The two most well-known cases are the case of $K\left(m_{1}, m_{2}\right)=\left(m_{1}+m_{2}\right) A$ (Safronov 1969; henceforth test 1) and the case of $K\left(m_{1}, m_{2}\right)=A$ (Smoluchowski 1916; henceforth test 2). In both cases $A$ is an arbitrary constant which we take to be $A=1$. Both tests were extensively discussed by Ohtsuki et al. (1990). They find that in particular for test 1 numerical algorithms generally deviate significantly from the analytic solution if the coordinate spacing in mass is too coarse $\left(m_{i+1} / m_{i} \gtrsim \sqrt{2}\right)$. This seems to be a particularly tough test case. In contrast they find that test 2 is much less challenging for numerical algorithms.

We have done both tests with our algorithm, following Ohtsuki's test procedure and using the analytic solutions presented in that paper. We find similar results as they find. For test 1 we get qualitative agreement, but the precise location of the peak of the distribution seems to depend on mass grid resolution and time step size. In Fig. C.1 the results are shown at a single given time, for four simulations: for 100 and 1000 grid points and for the largest possible time step $\left(\Delta t_{\max }=0.3 \max (f(\mathrm{~m}) / S(\mathrm{~m}))\right.$, where the factor 0.3 is our "safety parameter") and for 0.1 time that value. The modest but non-negligible deviations found in test 1 are slightly troubling. But since Ohtsuki et al. experience similar problems and the kernel of test 1 is very unlike the kernel we use in our models, we are reasonably satisfied with the results of these tests.

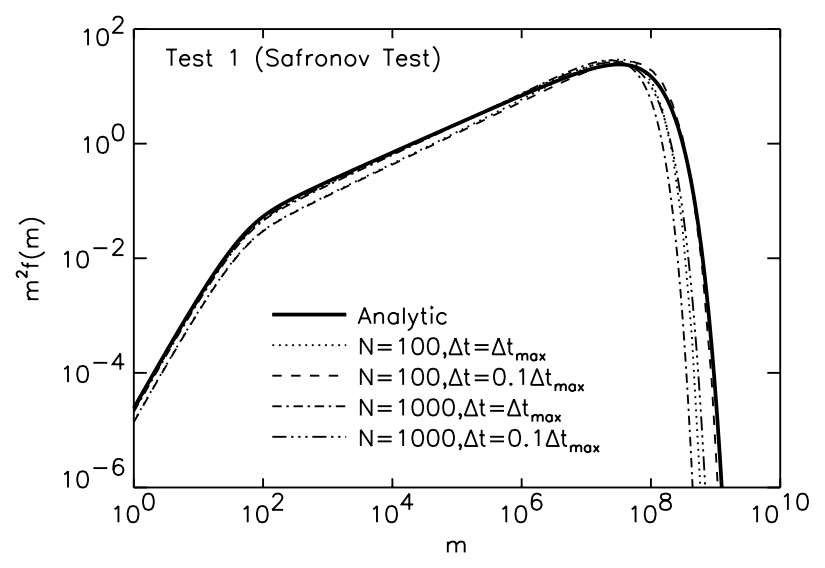

Fig. C.1. A snapshot of the distribution function of test 1 at dimensionless time $\tau=9$, for various values of the grid resolution and time step. The symbol $\Delta t$ is the time step $\left(\Delta t_{\max } \equiv \max (f(m) / S(m))\right)$ and $N$ is the number of grid points in $m\left(N=100\right.$ corresponds to $m_{i+1} / m_{i}=\sqrt{2}$, i.e. the highest resolution used by Ohtsuki; $N=1000$ corresponds to $\left.m_{i+1} / m_{i}=1.03517\right)$. The solid line is the analytic solution of Safronov.

For test 2 we get excellent agreement for moderate grid resolution (100 grid points) and for the largest possible time step size described above. The results are shown in Fig. C.2. Initially the difference between our model and the analytic solution is relatively large, but this is because our coagulation equation is formulated in a continuous form while the Smoluchowski solution is based on the discrete form of the equation. We therefore start with a different initial condition as in the analytic solution of Smoluchowski and logically we get initially somewhat different results. But as time progresses, the initial conditions are "forgotten", and the model result and the analytic solution start to agree better and better, ending with almost perfect agreement in our last time step.

These positive test results for simple kernels give hope that the full code, with dust settling, mixing and coagulation through Brownian motion and differential settling, also works well. Moreover, the stability and reliability of the code was confirmed by reproducing the results presented in this paper with different grid resolutions (both in $z$ and in $m$ ), and by using different time steps. But of course the only way to be sure that the code is working properly is a comparison of our code with various independent codes written by independent authors. Such a comparison has not been carried out. There is, however, an interesting way to test the code independently: by comparing the average size of grains rained out onto the midplane (in the absence of turbulence) to the result of the oneparticle model. From the one-particle model (Sect. 3) it follows that the size of the grain, once it reaches the midplane, is $a=0.01 \Sigma / 8 \rho_{\mathrm{d}}$ for compact grains. It should be kept in mind that this result was obtained by assuming that only the test particle rains down, while the other particles remain suspended in the disk. We can simulate this effect in the full code by taking two populations of grains: a fixed population (equal to the initial grain population) and an evolving population. The evolving population only grows by colliding with the fixed population, while the fixed population is not changing during the 


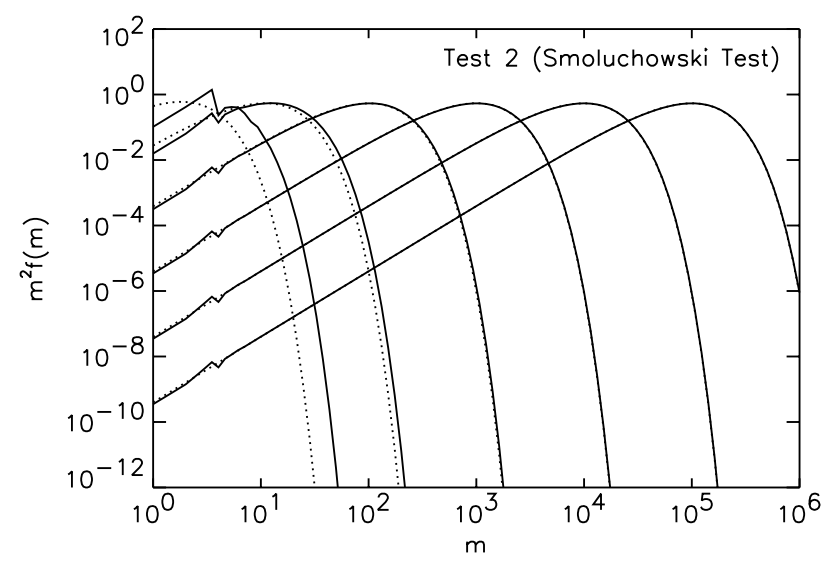

Fig. C.2. The time evolution of the distribution function in test 2 for the model (solid line) compared to the analytic solution of Smoluchowski (dotted line). The snapshots are at dimensionless time $\tau=1,10,10^{2}, 10^{3}, 10^{4}, 10^{5}$ from left to right. Due to different initial conditions the agreement is not good at $\tau=1$ but becomes better as time progresses because the initial conditions are "forgotten" by the system. The tiny wiggle in the solid curve around $m=4$ is a remainder of the initial conditions that apparently the algorithm does not "forget".

simulation. This does not conserve mass, but it does simulate the conditions similar to the one-particle model. We carried out this test for $\Sigma_{\text {gas }}=100 \mathrm{~g} / \mathrm{cm}^{2}$ at $R=1 \mathrm{AU}$ with $\rho_{\mathrm{d}}=3.6$, and we find that the model produces a sharp peak distribution near $a=0.35 \mathrm{~mm}$, which is virtually identical to the analytic result $(a=0.3472 \mathrm{~mm})$. That it is slightly larger is due the fact that the smallest grains still have a finite geometrical cross section.

\section{References}

Balbus, S., \& Hawley, J. 1991, ApJ, 376, 214

Blum, J., \& Wurm, G. 2000, Icarus, 143, 138

Bouwman, J., de Koter, A., van den Ancker, M. E., \& Waters, L. B. F. M. 2000, A\&A, 360, 213

Bouwman, J., de Koter, A., Dominik, C., \& Waters, L. B. F. M. 2003 , A\&A, 401, 577

Brearley, A. J., \& Jones, R. H. 1998, in Reviews in Mineralogy, 36, Planetary Materials, ed. J. J. Papike (Washington DC: Mineralogical Society of America)

Calvet, N., D'Alessio, P., Hartmann, L., et al. 2002, ApJ, 568, 1008

Cuzzi, J. N., Dobrovolskis, A. R., \& Champney, J. M. 1993, Icarus, 106, 102

Dominik, C., \& Tielens, A. 1995, PMA, 72, 783

Dominik, C., \& Tielens, A. 1996, PMA, 73, 1279

Dominik, C., \& Tielens, A. 1997, ApJ, 480, 647

Dubrulle, B., Morfill, G., \& Sterzik, M. 1995, Icarus, 114, 237

Dullemond, C. P., \& Dominik, C. 2004a, A\&A, 417, 159
Dullemond, C. P., \& Dominik, C. 2004b, A\&A, 421, 1075

Eisner, J. A., Lane, B. F., Akeson, R. L., Hillenbrand, L. A., \& Sargent, A. I. $2003, \mathrm{ApJ}, 588,360$

Gammie, C. F. 1996, ApJ, 457, 355

Goldreich, P., \& Ward, W. R. 1973, ApJ, 183, 1051

Honda, M., Kataza, H., Okamoto, Y. K., et al. 2003, ApJ, 585, L59

Klahr, H. H., \& Henning, T. 1997, Icarus, 128, 213

Lai, F. S., Friedlander, S., Pich, J., \& Hidy, G. M. 1972, J. Colloid Interface Sci., 39, 395

Leinert, C., van Boekel, R., Waters, L. B. F. M., et al. 2004, A\&A, 423, 537

Lissauer, J. J. 1993, ARA\&A, 31, 129

Malfait, K., Waelkens, C., Bouwman, J., De Koter, A., \& Waters, L. B. F. M. 1999, A\&A, 345, 181

Mathis, J. S., Rumpl, W., \& Nordsieck, K. H. 1977, ApJ, 217, 425

Meakin, P. 1987, CRC Critical Reviews in Solid State and Material Sciences, 13, 143

Meeus, G., Waters, L. B. F. M., Bouwman, J., et al. 2001, A\&A, 365, 476

Meeus, G., Sterzik, M., Bouwman, J., \& Natta, A. 2003, A\&A, 409, L25

Mizuno, H., Markiewicz, W. J., \& Voelk, H. J. 1988, A\&A, 195, 183

Ohtsuki, K. O., Nakagawa, Y., \& Nakazawa, K. 1990, Icarus, 83, 205

Ossenkopf, V. 1993, A\&A, 280, 617

Przygodda, F., van Boekel, R., Àbrahàm, P., et al. 2003, A\&A, 412, L43

Safronov, V. S. 1969, Evolution of the Protoplanetary Cloud and Formation of the Earth and Planets (Moskow: Nauka Press), in Russian (English translation: NASA TTF-677, 1972)

Schräpler, R., \& Henning, T. 2004, ApJ, 614, 960

Schumann, T. E. W. 1940, Quart. J. Roy. Meteorol. Soc., 66, 195

Smoluchowski, M. V. 1916, Zeitschrift fur Physik, 17, 557

Suttner, G., \& Yorke, H. W. 2001, ApJ, 551, 461

Suttner, G., Yorke, H. W., \& Lin, D. N. C. 1999, ApJ, 524, 857

Takeuchi, T., \& Lin, D. N. C. 2002, ApJ, 581, 1344

Testi, L., Natta, A., Shepherd, D. S., \& Wilner, D. J. 2003, A\&A, 403, 323

Todes, O. M. 1949, in Problemy kinetiki i kataliza (Problems of kinetics and Catalysis), Vol. VII, 137

van Boekel, R., Waters, L. B. F. M., Dominik, C., et al. 2003, A\&A, 400, L21

van Boekel, R., et al. 2004a, Nature, submitted

van Boekel, R., et al. 2004b, A\&A, submitted

van den Ancker, M. E., Bouwman, J., Wesselius, P. R., et al. 2000, A\&A, 357, 325

Voelk, H. J., Morfill, G. E., Roeser, S., \& Jones, F. C. 1980, A\&A, 85, 316

Weidenschilling, S. J. 1977, MNRAS, 180, 57

Weidenschilling, S. J. 1980, Icarus, 44, 172

Weidenschilling, S. J. 1984, Icarus, 60, 553

Weidenschilling, S. J. 1995, in Lunar and Planetary Institute Conference Abstracts, 1477

Weidenschilling, S. J. 1997a, Icarus, 127, 290

Weidenschilling, S. J. 1997b, in Lunar and Planetary Institute Conference, 28, 1517

Youdin, A. N., \& Shu, F. H. 2002, ApJ, 580, 494 